\title{
Some Fixed Point Theorems for Certain Contractive Mappings in G-Metric Spaces
}

\author{
Amit Singh, B. Fisher, And R.C. Dimri
}

\begin{abstract}
In this paper, we prove some fixed point theorems in complete G-metric spaces for self mappings satisfying different contractive conditions depended an another function. We also discuss that these mappings are G-continuous on such a fixed point.
\end{abstract}

\section{INTRODUCTION AND PRELIMINARIES}

In 1992, Dhage [1] introduced the concept of a $D$-metric space. The situation for a $D$-metric space is quite different from 2-metric spaces. Geometrically, a $D$-metric $D(x, y, z)$ represents the parameter of the triangle with vertices $x, y, z$ in $R^{2}$. Recently, Mustafa and Sims [3] showed that most of the results concerning Dhage's $D$-metric spaces are invalid. Therefore they introduced the improved version of the generalized metric space structure and called it a $G$-metric space. For more details of $G$-metric spaces, one can refer to the papers [2]-[11].

Now we give preliminaries and basic definitions which are helpful for proving our main results.

In 2004, Mustafa and Sims [4] introduced the concept of $G$-metric spaces as follows.

Definition 1. [4] Let $X$ be a nonempty set and let $G: X \times X \times X \rightarrow R^{+}$ be a function satisfying the following axioms:

$\left(G_{1}\right) G(x, y, z)=0$ if $x=y=z$,

$\left(G_{2}\right) 0<G(x, x, y)$, for all $x, y \in X$ with $x \neq y$,

$\left(G_{3}\right) G(x, x, y) \leq G(x, y, z)$, for all $x, y, z \in X$ with $z \neq y$,

$\left(G_{4}\right) G(x, y, z)=G(x, z, y)=G(y, z, x)=\ldots$ (symmetry in all three variables),

1991 Mathematics Subject Classification. Primary: 54H25; Secondary: 47H10.

Key words and phrases. G-metric spaces, fixed point, G-continuous, Contractive mappings, Depended function. 
$\left(G_{5}\right) G(x, y, z) \leq G(x, a, a)+G(a, y, z)$ for all $x, y, z, a \in X$ (rectangle inequality),

then the function $G$ is called a generalized metric, or, more specifically, a $G$-metric on $X$ and the pair $(X, G)$ is called a $G$-metric space.

Definition 2. [4] Let $(X, G)$ be a $G$-metric space and let $\left\{x_{n}\right\}$ be a sequence of points in $X$. Then a point $x$ in $X$ is said to be a limit of the sequence $\left\{x_{n}\right\}$ if $\lim _{m, n \rightarrow \infty} G\left(x, x_{n}, x_{m}\right)=0$ and then the sequence $\left\{x_{n}\right\}$ is said to be $G$-convergent to $x$. Thus, if $x_{n} \rightarrow x$ as $n \rightarrow \infty$, in a $G$-metric space $(X, G)$, then for each $\epsilon>0$, there exists a positive integer $N$ such that $G\left(x, x_{n}, x_{m}\right)<\epsilon$ for all $m, n, \in N$.

Proposition 1. [4] Let $(X, G)$ be a G-metric space. Then the following are equivalent

(i) $\left\{x_{n}\right\}$ is G-convergent to $x$,

(ii) $G\left(x_{n}, x_{n}, x\right) \rightarrow 0$ as $n \rightarrow \infty$,

(iii) $G\left(x_{n}, x, x\right) \rightarrow 0$ as $n \rightarrow \infty$,

(iv) $G\left(x_{m}, x_{n}, x\right) \rightarrow 0$ as $m, n \rightarrow \infty$.

Definition 3. [4] Let $(X, G)$ be a $G$-metric space. A sequence $\left\{x_{n}\right\}$ is called $G$-Cauchy if, for each $\epsilon>0$, there exists a positive integer $N$ such that $G\left(x_{n}, x_{m}, x_{\ell}\right)<\epsilon$ for all $n, m, \ell \in N$, i.e., $G\left(x_{n}, x_{m}, x_{\ell}\right) \rightarrow 0$ as $n, m, \ell \rightarrow \infty$.

Definition 4. $[4,5]$ Let $(X, G)$ and $\left(X^{\prime}, G^{\prime}\right)$ be two $G$-metric spaces and let $f:(X, G) \rightarrow\left(X^{\prime}, G^{\prime}\right)$ be a function, then $f$ is said to be $G$-continuous at a point $x_{0} \in X$ if given $\epsilon>0$, there exists $\delta>0$ such that for $x, y \in X$ and $G\left(x_{0}, x, y\right)<\delta$ implies $G^{\prime}\left(f\left(x_{0}\right), f(x), f(y)\right)<\epsilon$. A function $f$ is said to be $G$-continuous on $X$ if and only if it is $G$-continuous at all points $x_{0} \in X$. A function $f$ is said to be G-sequentially continuous at $x_{0}$, if whenever $\left\{x_{n}\right\}$ is $G$-convergent to $x_{0}$, then $\left\{f\left(x_{n}\right)\right\}$ is $G$-convergent to $f\left(x_{0}\right)$.

Proposition 2. [4] Let $(X, G)$ be a $G$-metric space. Then the function $G(x, y, z)$ is jointly continuous in all three of its variables.

Definition 5. [4] A $G$-metric space $(X, G)$ is called a symmetric $G$-metric space if $G(x, y, y)=G(y, x, x)$ for all $x, y \in X$.

Proposition 3. [4] Every $G$-metric space $(X, G)$ defines a metric space $\left(X, d_{G}\right)$ by putting

(i) $d_{G}(x, y)=G(x, y, y)+G(y, x, x)$ for all $x, y \in X$.

If $(X, G)$ is a symmetric $G$-metric space, then

(ii) $d_{G}(x, y)=2 G(x, y, y)$ for all $x, y \in X$.

However, if $(X, G)$ is not symmetric, then it follows from the $G$-metric properties that

(iii) $\frac{3}{2} G(x, y, y) \leq d_{G}(x, y) \leq G(x, y, y)$ for all $x, y \in X$. 
Definition 6. [4] A $G$-metric space $(X, G)$ is said to be $G$-complete if every $G$-Cauchy sequence in $(X, G)$ is $G$-convergent in $X$.

Proposition 4. [4] A G-metric space $(X, G)$ is said to be $G$-complete if and only if $\left(X, d_{G}\right)$ is a complete metric space.

Proposition 5. [4] Let $(X, G)$ be a $G$-metric space. Then, for any $x, y, z, a \in$ $X$, it follows that:

(i) if $G(x, y, z)=0$ then $x=y=z$,

(ii) $G(x, y, z) \leq G(x, x, y)+G(x, x, z)$

(iii) $G(x, y, y) \leq 2 G(y, y, x)$,

(iv) $G(x, y, z) \leq G(x, a, z)+G(a, y, z)$,

(v) $G(x, y, z) \leq \frac{2}{3}(G(x, y, a)+G(x, a, z)+G(a, y, z))$,

(vi) $G(x, y, z) \leq G(x, a, a)+G(y, a, a)+G(z, a, a)$.

Lemma 1. [11] Let $(X, G)$ be a $G$-metric space and $T$ be a self map on $X$ satisfying

$$
G(T x, T y, T z) \leq q G(x, y, z)
$$

for all $x, y, z \in X$, where $0 \leq q<1$ and $x_{n}=T x_{n-1}=T\left(T x_{n-2}\right)=\cdots=$ $T^{n}\left(x_{0}\right)$ for some $x_{0} \in X$, then $\left\{x_{n}\right\}$ is a G-Cauchy sequence in $X$.

\section{MAin RESUlts}

Theorem 1. Let $(X, G)$ be a complete $G$-metric space and let $S, T: X \rightarrow X$ such that $T$ is one-to-one and satisfy

$$
\begin{aligned}
& G(T S x, T S y, T S z) \leq \\
& k \max \left\{\begin{array}{l}
G(T x, T S x, T S x), G(T y, T S y, T S y), \\
G(T y, T S z, T S z), G(T z, T S z, T S z), G(T z, T S y, T S y), \\
\quad \frac{G(T x, T S y, T S y)+G(T y, T S x, T S x)}{2}, \\
\left.\quad \frac{G(T x, T S z, T S z)+G(T z, T S x, T S x)}{2}\right\}
\end{array}\right.
\end{aligned}
$$

for all $x, y, z \in X$ and $0 \leq k<1$. Then $S$ has a unique common fixed point and $S$ is $G$-continuous at the fixed point.

Proof. Let $x_{0}$ be an arbitrary point in $X$. We define the iterative sequence $\left\{x_{n}\right\}$ by $x_{n+1}=S x_{n}=S^{n+1} x_{0}$ for $n=0,1,2, \ldots$

Putting $x=x_{n}, y=x_{n+1}$ and $z=x_{n+1}$ in (3), we have 
(3)

$$
\begin{aligned}
& G\left(T x_{n}, T x_{n+1}, T x_{n+1}\right)=G\left(T S x_{n-1}, T S x_{n}, T S x_{n}\right) \\
& \leq k \max \left\{G\left(T x_{n-1}, T S x_{n-1}, T S x_{n-1}\right), G\left(T x_{n}, T S x_{n}, T S x_{n}\right)\right. \text {, } \\
& G\left(T x_{n}, T S x_{n}, T S x_{n}\right), G\left(T x_{n}, T S x_{n}, T S x_{n}\right), \\
& G\left(T x_{n}, T S x_{n}, T S x_{n}\right) \text {, } \\
& \frac{G\left(T x_{n-1}, T S x_{n}, T S x_{n}\right)+G\left(T x_{n}, T S x_{n-1}, T S x_{n-1}\right)}{2}, \\
& \left.\frac{G\left(T x_{n-1}, T S x_{n}, T S x_{n}\right)+G\left(T x_{n}, T S x_{n-1}, T S x_{n-1}\right)}{2}\right\} \\
& \leq k \max \left\{G\left(T x_{n-1}, T x_{n}, T x_{n}\right), G\left(T x_{n}, T x_{n+1}, T x_{n+1}\right),\right. \\
& G\left(T x_{n}, T x_{n+1}, T x_{n+1}\right), G\left(T x_{n}, T x_{n+1}, T x_{n+1}\right), \\
& G\left(T x_{n}, T_{n+1}, T x_{n+1}\right) \text {, } \\
& \frac{G\left(T x_{n-1}, T x_{n+1}, T x_{n+1}\right)+G\left(T x_{n}, T x_{n}, T x_{n}\right)}{2}, \\
& \left.\frac{G\left(T x_{n-1}, T x_{n+1}, T x_{n+1}\right)+G\left(T x_{n}, T x_{n}, T x_{n}\right)}{2}\right\} \\
& =k \max \left\{G\left(T x_{n-1}, T x_{n}, T x_{n}\right), G\left(T x_{n}, T x_{n+1}, T x_{n+1}\right)\right\},
\end{aligned}
$$

since

$$
\begin{aligned}
G\left(T x_{n-1}, T x_{n+1}, T x_{n+1}\right) & \leq G\left(T x_{n-1}, T x_{n}, T x_{n}\right)+G\left(T x_{n}, T x_{n+1}, T x_{n+1}\right) \\
& \leq 2 G\left(T x_{n-1}, T x_{n}, T x_{n}\right) .
\end{aligned}
$$

It follows from (3) that

$$
G\left(T x_{n}, T x_{n+1}, T x_{n+1}\right) \leq k G\left(T x_{n-1}, T x_{n}, T x_{n}\right) .
$$

and by Lemma 1 , we have $\left\{T x_{n}\right\}$ is a $G$-Cauchy sequence in $X$. Since $(X, G)$ is a complete $G$-metric space, there exists $u \in X$ such that $T x_{n} \rightarrow u$.

Suppose that $T S(u) \neq T u$. Then by using (2), we have

$$
\begin{aligned}
& G\left(T x_{n}, T S u, T S u\right)=G\left(T S x_{n-1}, T S u, T S u\right) \\
& \leq k \max \left\{G\left(T x_{n-1}, T S x_{n-1}, T S x_{n-1}\right), G(T u, T S u, T S u),\right. \\
& G(T u, T S u, T S u), G(T u, T S u, T S u), G(T u, T S u, T S u), \\
& \frac{G\left(T x_{n-1}, T S u, T S u\right)+G\left(T u, T S x_{n-1}, T S x_{n-1}\right)}{2}, \\
& \left.\frac{G\left(T x_{n-1}, T S u, T S u\right)+G\left(T u, T S x_{n-1}, T S x_{n-1}\right)}{2}\right\}=
\end{aligned}
$$




$$
\begin{aligned}
& =k \max \left\{G\left(T x_{n-1}, T x_{n}, T x_{n}\right), G(T u, T S u, T S u),\right. \\
& G(T u, T S u, T S u), G(T u, T S u, T S u), \\
& G(T u, T S u, T S u) \text {, } \\
& \frac{G\left(T x_{n-1}, T S u, T S u\right)+G\left(T u, T x_{n}, T x_{n}\right)}{2}, \\
& \left.\frac{G\left(T x_{n-1}, T S u, T S u\right)+G\left(T u, T x_{n}, T x_{n}\right)}{2}\right\} \\
& =k \max \left\{G\left(T x_{n-1}, T x_{n}, T x_{n}\right), G\left(T x_{n-1}, T S u, T S u\right)\right. \text {, } \\
& \left.G\left(T u, T x_{n}, T x_{n}\right), G(T u, T S u, T S u)\right\} .
\end{aligned}
$$

Taking the limit as $n \rightarrow \infty$ and using the fact that $G$ is a continuous function in its variables, we obtain

$$
G(T u, T S u, T S u) \leq k G(T u, T S u, T S u)
$$

a contradiction as $0 \leq k<1$. Hence $T S u=T u$. Since $T$ is one-to-one, we have $S u=u$ and so $u$ is a fixed point of $S$.

To prove its uniqueness, suppose that $v$ is a second distinct fixed point of $S$. Then from the injectivity of $T$ we get $S u=S v$, a contradiction. Hence the fixed point is unique.

Now let $\left\{y_{n}\right\}$ be any sequence in $X$ such that $\lim _{n \rightarrow \infty} T y_{n}=T u$. Then by using (2), we have

$$
\begin{gathered}
G\left(T S y_{n}, T S u, T S y_{n}\right) \leq \\
\leq k \max \left\{G\left(T y_{n}, T S y_{n}, T S y_{n}\right), G(T u, T S u, T S u),\right. \\
G\left(T u, T S y_{n}, T S y_{n}\right), G\left(T y_{n}, T S y_{n}, T S y_{n}\right), \\
G\left(T y_{n}, T S u, T S u\right), \\
\frac{G\left(T y_{n}, T S u, T S u\right)+G\left(T u, T S y_{n}, T S y_{n}\right)}{2}, \\
\left.\frac{G\left(T x, T S y_{n}, T S y_{n}\right)+G\left(T y_{n}, T S y_{n}, T S y_{n}\right)}{2}\right\} \\
=k \max \left\{G\left(T y_{n}, T S y_{n}, T S y_{n}\right), G\left(T y_{n}, T S u, T S u\right),\right. \\
\left.G(T u, T S u, T S u), G\left(T u, T S y_{n}, T S y_{n}\right)\right\} .
\end{gathered}
$$


This reduces to

$$
\begin{aligned}
G\left(T S y_{n}, T u, T S y_{n}\right) \leq k \max \left\{G\left(T y_{n}, T S y_{n}, T S y_{n}\right),\right. \\
\\
\left.G\left(T y_{n}, T u, T u\right), G\left(T u, T S y_{n}, T S y_{n}\right)\right\} \\
=k \max \left\{G\left(T y_{n}, T S y_{n}, T S y_{n}\right), G\left(T y_{n}, T u, T u\right)\right\} .
\end{aligned}
$$

Taking the limit as $n \rightarrow \infty$, we have $G\left(T u, T S y_{n}, T S y_{n}\right) \rightarrow 0$ and by definition of $G$-continuity of a $G$-metric space $(X, G)$, we have $T S y_{n} \rightarrow T u=$ $T S u$, since $T$ is one-to-one. Therefore $S y_{n} \rightarrow u=S u$ which implies that $S$ is $G$-continuous at $u$.

Corollary 1. Let $(X, G)$ be a complete $G$-metric space and let $S, T: X \rightarrow X$ such that $T$ is one-to-one and satisfies

$$
\begin{aligned}
& G\left(T^{m} S^{m} x, T^{m} S^{m} y, T^{m} S^{m} z\right) \leq \\
& \leq k \max \left\{G\left(T^{m} x, T^{m} S^{m} x, T^{m} S^{m} x\right), G\left(T^{m} y, T^{m} S^{m} y, T^{m} S^{m} y\right)\right. \text {, } \\
& G\left(T^{m} y, T^{m} S^{m} z, T^{m} S^{m} z\right), G\left(T^{m} z, T^{m} S^{m} z, T^{m} S^{m} z\right), \\
& G\left(T^{m} z, T^{m} S^{m} y, T^{m} S^{m} y\right) \text {, } \\
& \frac{G\left(T^{m} x, T^{m} S^{m} y, T^{m} S^{m} y\right)+G\left(T^{m} y, T^{m} S^{m} x, T^{m} S^{m} x\right)}{2}, \\
& \left.\frac{G\left(T^{m} x, T^{m} S^{m} z, T^{m} S^{m} z\right)+G\left(T^{m} z, T^{m} S^{m} x, T^{m} S^{m} x\right)}{2}\right\}
\end{aligned}
$$

for all $x, y, z \in X, m \in N$ and $0 \leq k<1$. Then $S$ has a unique common fixed point and $S^{m}$ is $G$-continuous at the fixed point.

Proof. On the lines of Theorem 1, one can easily see that $S^{m}$ has a unique fixed point, say $u$, and that $S^{m}$ is $G$-continuous at $u$. But $S u=S\left(S^{m} u\right)=$ $S^{m+1} u=S^{m}(S u)$, and so $S u$ is another fixed point of $S^{m}$. Thus, by the uniqueness we have $S u=u$ and so $u$ is the unique fixed point of $S$.

Theorem 2. Let $(X, G)$ be a complete $G$-metric space and let $S, T: X \rightarrow X$ such that $T$ is one-to-one and satisfies

(6)

$$
\begin{aligned}
& G(T S x, T S y, T S z) \leq \\
& \leq k \max \left\{\begin{array}{l}
G(T x, T S x, T S x)+G(T y, T S y, T S y)+G(T z, T S z, T S z), \\
G(T x, T S y, T S y)+G(T y, T S x, T S x)+G(T z, T S y, T S y), \\
G(T x, T S z, T S z)+G(T y, T S z, T S z)+G(T z, T S x, T S x)\}
\end{array}\right.
\end{aligned}
$$

for all $x, y, z \in X$ and $0 \leq k<\frac{1}{4}$. Then $S$ has a unique common fixed point and $S$ is $G$-continuous at the fixed point. 
Proof. Let $x_{0}$ be an arbitrary point in $X$. We define the iterative sequence $\left\{x_{n}\right\}$ by $x_{n+1}=S x_{n}=S^{n+1} x_{0}, n=0,1,2, \ldots$

We now prove that $\left\{x_{n}\right\}$ is a $G$-Cauchy sequence in $X$. Let $x=x_{n}$, $y=x_{n+1}$ and $z=x_{n+1}$ in (6). We then have

$$
\begin{aligned}
& G\left(T x_{n}, T x_{n+1}, T x_{n+1}\right)=G\left(T S x_{n-1}, T S x_{n}, T S x_{n}\right) \\
& \leq k \max \left\{G\left(T x_{n-1}, T S x_{n-1}, T S x_{n-1}\right)+G\left(T x_{n}, T S x_{n}, T S x_{n}\right)+\right. \\
& G\left(T x_{n}, T S x_{n}, T S x_{n}\right), G\left(T x_{n-1}, T S x_{n}, T S x_{n}\right)+ \\
& G\left(T x_{n}, T S x_{n-1}, T S x_{n-1}\right)+G\left(T x_{n}, T S x_{n}, T S x_{n}\right), \\
& G\left(T x_{n-1}, T S x_{n}, T S x_{n}\right)+G\left(T x_{n}, T S x_{n}, T S x_{n}\right)+ \\
& \left.G\left(T x_{n}, T S x_{n-1}, T S x_{n-1}\right)\right\} \\
& =k \max \left\{G\left(T x_{n-1}, T x_{n}, T x_{n}\right)+G\left(T x_{n}, T x_{n+1}, T x_{n+1}\right)+\right. \\
& G\left(T x_{n}, T x_{n+1}, T x_{n+1}\right), G\left(T x_{n-1}, T x_{n+1}, T x_{n+1}\right)+ \\
& G\left(T x_{n}, T x_{n}, T x_{n}\right)+G\left(T x_{n}, T x_{n+1}, T x_{n+1}\right), \\
& G\left(T x_{n-1}, T x_{n+1}, T x_{n+1}\right)+G\left(T x_{n}, T x_{n+1}, T x_{n+1}\right)+ \\
& \left.G\left(T x_{n}, T x_{n}, T x_{n}\right)\right\} \\
& =k \max \left\{G\left(T x_{n-1}, T x_{n}, T x_{n}\right)+2 G\left(T x_{n}, T x_{n+1}, T x_{n+1}\right),\right. \\
& G\left(T x_{n-1}, T x_{n+1}, T x_{n+1}\right)+G\left(T x_{n}, T x_{n+1}, T x_{n+1}\right), \\
& \left.G\left(T x_{n-1}, T x_{n+1}, T x_{n+1}\right)+G\left(T x_{n}, T x_{n+1}, T x_{n+1}\right)\right\} \\
& =k \max \left\{G\left(T x_{n-1}, T x_{n}, T x_{n}\right)+2 G\left(T x_{n}, T x_{n+1}, T x_{n+1}\right),\right. \\
& \left.G\left(T x_{n-1}, T x_{n+1}, T x_{n+1}\right)+G\left(T x_{n}, T x_{n+1}, T x_{n+1}\right)\right\} .
\end{aligned}
$$

Case 1. Suppose that

$$
\begin{aligned}
& \max \left\{G\left(T x_{n-1}, T x_{n}, T x_{n}\right)+2 G\left(T x_{n}, T x_{n+1}, T x_{n+1}\right),\right. \\
& \left.G\left(T x_{n-1}, T x_{n+1}, T x_{n+1}\right)+G\left(T x_{n}, T x_{n+1}, T x_{n+1}\right)\right\}= \\
& =G\left(T x_{n-1}, T x_{n}, T x_{n}\right)+2 G\left(T x_{n}, T x_{n+1}, T x_{n+1}\right) .
\end{aligned}
$$

Then, using (7), we get

$G\left(T x_{n}, T x_{n+1}, T x_{n+1}\right) \leq k\left\{G\left(T x_{n-1}, T x_{n}, T x_{n}\right)+2 G\left(T x_{n}, T x_{n+1}, T x_{n+1}\right)\right\}$ and so

$$
G\left(T x_{n}, T x_{n+1}, T x_{n+1}\right) \leq q\left\{G\left(T x_{n-1}, T x_{n}, T x_{n}\right)\right\},
$$

where $q=\frac{k}{1-2 k}$ and $q<1$, as $0 \leq k \leq \frac{1}{4}$. Thus by Lemma 12, we have $\left\{T x_{n}\right\}$ is a $G$-Cauchy sequence in $X$. 
Case 2. Suppose that

$$
\begin{gathered}
\max \left\{G\left(T x_{n-1}, T x_{n}, T x_{n}\right)+2 G\left(T x_{n}, T x_{n+1}, T x_{n+1}\right),\right. \\
\left.G\left(T x_{n-1}, T x_{n+1}, T x_{n+1}\right)+G\left(T x_{n}, T x_{n+1}, T x_{n+1}\right)\right\}= \\
=G\left(T x_{n-1}, T x_{n+1}, T x_{n+1}\right)+G\left(T x_{n}, T x_{n+1}, T x_{n+1}\right) .
\end{gathered}
$$

Then (7) reduces to

$$
\begin{aligned}
& G\left(T x_{n}, T x_{n+1}, T x_{n+1}\right) \leq \\
& \leq k\left\{G\left(T x_{n-1}, T x_{n+1}, T x_{n+1}\right)+G\left(T x_{n}, T x_{n+1}, T x_{n+1}\right)\right\} .
\end{aligned}
$$

Now using $\mathrm{G}_{5}$ of Definition 1 , we have

$$
\begin{aligned}
& G\left(T x_{n-1}, T x_{n+1}, T x_{n+1}\right) \leq \\
& G\left(T x_{n-1}, T x_{n}, T x_{n}\right)+G\left(T x_{n}, T x_{n+1}, T x_{n+1}\right) .
\end{aligned}
$$

Now (8) becomes

$$
G\left(T x_{n}, T x_{n+1}, T x_{n+1}\right) \leq q\left\{G\left(T x_{n-1}, T x_{n}, T x_{n}\right)\right\}
$$

where again $q=\frac{k}{1-2 k}$ and $q<1$. Thus, by Lemma 12 , we have $\left\{T x_{n}\right\}$ is a $G$-Cauchy sequence in $X$.

Hence in all cases, the sequence $\left\{T x_{n}\right\}$ is a $G$-Cauchy sequence in $X$ and since $(X, G)$ is a complete $G$-metric space, there exists $u \in X$ such that $T x_{n} \rightarrow u$.

Suppose, if possible, that $T S(u) \neq T u$. Then by using (6), we have

$$
\begin{aligned}
& G\left(T x_{n}, T S u, T S u\right)=G\left(T S x_{n-1}, T S u, T S u\right) \\
& \leq k \max \left\{G\left(T x_{n-1}, T S x_{n-1}, T S x_{n-1}\right)+G(T u, T S u, T S u)+\right. \\
& G(T u, T S u, T S u), G\left(T x_{n-1}, T S u, T S u\right)+ \\
& G\left(T u, T S x_{n-1}, T S x_{n-1}\right)+G(T u, T S u, T S u) \\
& G\left(T x_{n-1}, T S u, T S u\right)+G(T u, T S u, T S u), \\
& \left.G\left(T u, T S x_{n-1}, T S x_{n-1}\right)\right\}=
\end{aligned}
$$




$$
\begin{gathered}
=k \max \left\{G\left(T x_{n-1}, T x_{n}, T x_{n}\right)+G(T u, T S u, T S u)+\right. \\
G(T u, T S u, T S u), G\left(T x_{n-1}, T S u, T S u\right)+ \\
G\left(T u, T x_{n}, T x_{n}\right)+G(T u, T S u, T S u) \\
G\left(T x_{n-1}, T S u, T S u\right)+G(T u, T S u, T S u), \\
\left.G\left(T u, T x_{n}, T x_{n}\right)\right\} \\
=k \max \left\{G\left(T x_{n-1}, T x_{n}, T x_{n}\right)+2 G(T u, T S u, T S u)\right. \\
G\left(T x_{n-1}, T S u, T S u\right)+G\left(T u, T x_{n}, T x_{n}\right)+ \\
G(T u, T S u, T S u)\} .
\end{gathered}
$$

Taking the limit as $n \rightarrow \infty$ and using the fact that $G$ is a continuous function in its variables, we obtain

$$
\begin{aligned}
G(T u, T S u, T S u) & \leq k \max \{2 G(T u, T S u, T S u), 2 G(T u, T S u, T S u)\} \\
& \leq 2 k G(T u, T S u, T S u),
\end{aligned}
$$

a contradiction since $0 \leq k \leq \frac{1}{4}$. Hence $T S u=T u$ and since $T$ is one-to-one, we have $S u=u$. Hence $u$ is a fixed point of $S$.

To prove its uniqueness, suppose that $v$ is a second fixed point of $S$. Then by (6), we have

$$
\begin{gathered}
G(T u, T v, T v)=G(T S u, T S v, T S v) \\
\leq k \max \{G(T u, T u, T u)+G(T v, T v, T v)+G(T v, T v, T v) \\
G(T u, T v, T v)+G(T v, T u, T u)+G(T v, T v, T v) \\
G(T u, T v, T v)+G(T v, T v, T v)+G(T v, T u, T u)\} \\
=k \max \{G(T u, T v, T v)+G(T v, T u, T u)\}
\end{gathered}
$$

and so

$$
G(T u, T v, T v) \leq q G(T v, T u, T u) .
$$

Now, by the same argument, we obtain

$$
G(T v, T u, T u) \leq q G(T u, T v, T v)
$$

and so

$$
G(T u, T v, T v) \leq q^{2} G(T v, T u, T u),
$$

a contradiction, since $q<1$. Therefore $u=v$, proving the uniqueness.

Now let $\left\{y_{n}\right\}$ be any sequence in $X$ such that $\lim _{n \rightarrow \infty} T y_{n}=T u$, then by using (6), we have 


$$
\begin{gathered}
G\left(T S y_{n}, T S u, T S u\right) \\
\leq k \max \left\{\begin{array}{c}
G\left(T y_{n}, T S y_{n}, T S y_{n}\right)+G(T u, T S u, T S u)+ \\
G(T u, T S u, T S u) \\
G\left(T y_{n}, T S u, T S u\right)+G\left(T u, T S y_{n}, T S y_{n}\right)+ \\
G(T u, T S u, T S u) \\
G\left(T y_{n}, T S u, T S u\right)+G(T u, T S u, T S u)+ \\
\left.G\left(T u, T S y_{n}, T S y_{n}\right)\right\} \\
=k \max \left\{G\left(T y_{n}, T S y_{n}, T S y_{n}\right)+2 G(T u, T S u, T S u)\right. \\
G\left(T y_{n}, T S u, T S u\right)+G(T u, T S u, T S u)+ \\
\left.G\left(T u, T S y_{n}, T S y_{n}\right)\right\} .
\end{array}\right.
\end{gathered}
$$

Case 1: if

$$
\begin{aligned}
& \max \left\{G\left(T y_{n}, T S y_{n}, T S y_{n}\right)+2 G(T u, T S u, T S u)+\right. \\
& \left.G\left(T y_{n}, T S u, T S u\right)+G(T u, T S u, T S u)+G\left(T u, T S y_{n}, T S y_{n}\right)\right\} \\
& =\left\{G\left(T y_{n}, T S y_{n}, T S y_{n}\right)+2 G(T u, T S u, T S u)\right\} .
\end{aligned}
$$

Then (10) reduces to

$$
\begin{aligned}
& G\left(T S y_{n}, T S u, T S u\right) \leq \\
& \quad k \max \left\{G\left(T y_{n}, T S y_{n}, T S y_{n}\right)+2 G(T u, T S u, T S u)\right\} .
\end{aligned}
$$

Letting limit $n \rightarrow \infty$ and using $\mathrm{TSu}=\mathrm{Tu}$ and $\mathrm{T} y_{n} \rightarrow T u$, we have

$$
\begin{aligned}
G\left(T S y_{n}, T u, T u\right) & \leq k \max \left\{G\left(T u, T S y_{n}, T S y_{n}\right)+2 G(T u, T u, T u)\right\} \\
& =k G\left(T u, T S y_{n}, T S y_{n}\right) .
\end{aligned}
$$

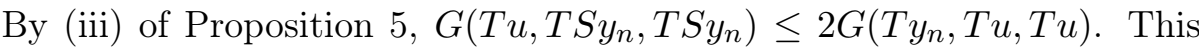
implies that (11) reduces to $G\left(T S y_{n}, T u, T u\right) \leq 0$. But $G\left(T S y_{n}, T u, T u\right) \geq$ 0 . Hence $G\left(T S y_{n}, T u, T u\right)=0$. So $T S y_{n} \rightarrow T u=T S u$. Since $T$ is one-toone, therefore $S y_{n} \rightarrow u=S u$.

This implies that $S$ is G-continuous at $u$. 
Case 2: If

$$
\begin{gathered}
\max \left\{G\left(T y_{n}, T S y_{n}, T S y_{n}\right)+2 G(T u, T S u, T S u)\right. \\
\left.G\left(T y_{n}, T S u, T S u\right)+G(T u, T S u, T S u)+G\left(T u, T S y_{n}, T S y_{n}\right)\right\} \\
=\left\{G\left(T y_{n}, T S u, T S u\right)+G(T u, T S u, T S u)+G\left(T u, T S y_{n}, T S y_{n}\right)\right\} .
\end{gathered}
$$

Then (2.9) reduces to

$$
\begin{aligned}
G\left(T S y_{n}, T S u, T S u\right) \leq k \max & \left\{G\left(T y_{n}, T S u, T S u\right)+G(T u, T S u, T S u)\right. \\
& \left.+G\left(T u, T S y_{n}, T S y_{n}\right)\right\} .
\end{aligned}
$$

Letting limit $n \rightarrow \infty$ and using $T S u=T u$ and $T y_{n} \rightarrow T u$, we have

$$
\begin{aligned}
& G\left(T S y_{n}, T u, T u\right) \leq k \max \{G(T u, T u, T u)+G(T u, T u, T u) \\
&\left.+G\left(T u, T S y_{n}, T S y_{n}\right)\right\} \\
&=K G\left(T u, T S y_{n}, T S y_{n}\right) .
\end{aligned}
$$

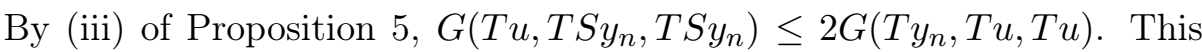
implies that (12) reduces to $G\left(T S y_{n}, T u, T u\right) \leq 0$. But $G\left(T S y_{n}, T u, T u\right) \geq$ 0 .

Hence $G\left(T S y_{n}, T u, T u\right)=0$, so $T S y_{n} \rightarrow T u=T S u$. Since $T$ is one-toone. Therefore $S y_{n} \rightarrow u=S u$.

This implies that $S$ is G-continuous at $u$.

Therefore in both cases $S$ is G-continuous at point $u$. Hence completes the theorem.

Corollary 2. Let $(X, G)$ be a complete $G$-metric space and let $S, T: X \rightarrow X$ such that $T$ is one-to-one and satisfying

$$
\begin{aligned}
& G\left(T^{m} S^{m} x, T^{m} S^{m} y, T^{m} S^{m} z\right) \leq \\
& \leq k \max \left\{G\left(T^{m} x, T^{m} S^{m} x, T^{m} S^{m} x\right)+G\left(T^{m} y, T^{m} S^{m} y, T^{m} S^{m} y\right)\right. \\
&+ G\left(T^{m} z, T^{m} S^{m} z, T^{m} S^{m} z\right), G\left(T^{m} x, T^{m} S^{m} y, T^{m} S^{m} y\right)+ \\
& G\left(T^{m} y, T^{m} S^{m} x, T^{m} S^{m} x\right)+G\left(T^{m} z, T^{m} S^{m} y, T^{m} S^{m} y\right), \\
& G\left(T^{m} x, T^{m} S^{m} z, T^{m} S^{m} z\right)+G\left(T^{m} y, T^{m} S^{m} z, T^{m} S^{m} z\right)+ \\
&\left.G\left(T^{m} z, T^{m} S^{m} x, T^{m} S^{m} x\right)\right\} .
\end{aligned}
$$

for all $x, y, z \in X, m \in N$ and $0 \leq k<\frac{1}{4}$. Then $S$ has a unique common fixed point and $S^{m}$ is G-continuous at the fixed point.

Proof. On the lines of Theorem 2, one can easily obtain, $S^{m}$ has a unique fixed point say u, i.e., $S^{m} u=u$ and $S^{m}$ is G-continuous at $u$. 
But $S u=S\left(S^{m} u\right)=S^{m+1} u=S^{m}(S u)$, so $S u$ is another fixed point of $S^{m}$. By uniqueness $S u=u$, i.e., $u$ is a unique fixed point of $S$.

Acknowledgement. The authors are very grateful to the editor and the reviewers for their valuable suggestions which lead to the improvement of the paper. The first author also would like to express his deep thanks to second author Professor B. Fisher for his advice during the preparation of the revised version of the paper.

\section{REFERENCES}

[1] Dhage, B.C., Generalized metric space and mappings with fixed point, Bull. Cal. Math. Soc., 84(1992), 329-336.

[2] Manro, S., Kumar, S., Bhatia, S.S., R-weakly commuting maps in G-metric spaces, Fasc. Math. Nr. 47 (2011), 11-17.

[3] Mustafa Z, Sims, B., Some remarks concerning D-metric spaces, Proceeding of International Conference on Fixed Point Theory and Applications, Yokohama Publishers, Valencia Spain, July 13-19, 2004, 189-198.

[4] Mustafa Z, Sims, B., A new approach to a generalized metric spaces, J. Nonlinear Convex Anal., 7 (2006), 289-297.

[5] Mustafa Z, Sims, B., Fixed point theorems for contractive mappings in complete Gmetric spaces, Fixed Point Theory and Applications, Vol. 2009, Article ID 917175, 10 pages.

[6] Mustafa, Z., Obiedat, H., Awawdeh, F., Some fixed point theorems for mappings on complete G-metric spaces, Fixed Point Theory and Applications, Vol. 2008, Article ID 18970, 12 pages.

[7] Mustafa, Z., Shatanawi, W, Bataineh, M., Existence of fixed point results in G-metric spaces, International Journal of Mathematics and Mathematical Sciences, Vol. 2009, Article ID 283028, 10 pages.

[8] Mustafa, Z., Obiedat, H., A fixed point theorem of Riech in G-metric spaces, Cubo a Mathematics Journal, 12 (1) (2010), 83-93.

[9] Mustafa, Z., Awawdeh, F., Shatanawi, W, Fixed point theorems for expansive mappings in G-metric spaces, Int. J. Contemp. Math. Sci., 5 (50 (2010), 2463-2472.

[10] Shatanawi, W, Fixed point theory for contractive mappings satisfying $\phi$-maps in Gmetric spaces, Fixed Point Theory and Applications, Vol. 2010, Article ID 181650, 9 pages.

[11] Vats, R.K., Kumar, S., Sihag, V., Fixed point theorems in complete G-metric space, Fasc. Math., Nr. 47 (2011), 127-139. 


\section{Amit Singh}

Department of Mathematics

Government Degree College Billawar

JAMMU AND KASHMIR-184204

INDIA

E-mail address: singhamit841@gmail.com

\section{B. Fisher}

Department Of Mathematics

UNIVERSITY OF LEICESTER

LEICESTER, LE1 7RH

ENGLAND

E-mail address: fbr@le.ac.uk

R.C. Dimri

Department of Mathematics

H.N.B. Garhwal University

Srinagar (GARHWAL), UtTarakHand-246174

INDIA

E-mail address: dimrirc@gmail.com 